\title{
Shedding light on maternal sunlight exposure during pregnancy and considerations for public health policy
}

\author{
Peter Anto Johnson*, John Christy Johnson
}

Faculty of Medicine and Dentistry, University of Alberta, Edmonton, AB, Canada

*Author for correspondence (paj1@ualberta.ca)

\begin{abstract}
:
Sunlight exposure during pregnancy could be implicated in the physiological development and growth of the fetus, as well as long-term health after birth. Although several studies suggest the involvement of ultraviolet radiation-mediated vitamin D synthesis, current knowledge regarding the effects of sunlight exposure during pregnancy remains limited. We aimed to (i) summarize the existing body of research studying the influence of sunlight exposure on fetal growth-related birth outcomes and longterm health outcomes and (ii) determine its implications for therapeutics and public health policy. Of the studies identified on birth outcomes, the majority (5/8) demonstrated an association between sunlight exposure and reduced adverse birth outcomes (e.g., low birth weight, preterm births, small for gestational age, etc.), 2/8 studies showed no association, and 1/8 suggested a negative association between sunlight exposure and reduction of these adverse birth outcomes. Of the studies examining long-term health outcomes, sunlight exposure during pregnancy was shown to promote skeletal growth and development $(2 / 6)$, and reduce the incidence of multiple sclerosis (2/6), asthma (2/6) and pneumonia (1/6). However, several of these studies used different methodologies and populations making it difficult to compare and integrate findings. Based on these results, we examined: the importance of exposure at different stages of pregnancy, proposed mechanisms by which sunlight exposure could lead to optimal outcomes, epidemiological differences influencing the findings, and necessary practical considerations prior to the implementation of public health policy recommendations. While these findings are promising, more rigorous research is warranted to support these recommendations.
\end{abstract}

\section{Introduction}

Sunlight consists of infrared, visible, and ultraviolet (UV) radiation and plays a critical role in regulating normal physiological functions, including vitamin $\mathrm{D}$ production and synthesis of hormones such as serotonin in the brain $[1,2]$; however, little is understood about its effects during pregnancy on offspring. Exposure to environmental stressors during critical periods of growth and development of the offspring could have severe consequences on its short- and long-term health [3], suggesting that differences in level of exposure to sunlight during pregnancy could considerably impact immediate and long-term outcomes. This is highly relevant in both developing regions with abundant sunlight exposure, as well as in regions, such as Canada, where greater seasonal differences result in a lower amount of exposure to sunlight for several months of the year.

Overexposure to UV rays during pregnancy is associated with increased adverse risks in mothers including skin damage and cancers [4]. At the same time, exposure to $\mathrm{UV}$ is essential for chemical reactions involved in conversion pathways of cholesterol to vitamin $\mathrm{D}$ for several functional roles; these include calcium and phosphate homeostasis, insulin metabolism, bone growth, and remodeling, cell growth mediation and promotion of immune function [2]. Pregnant women are increasingly more susceptible to vitamin D deficiency [5], suggesting there is a greater demand for vitamin D in the body during pregnancy. Maternal vitamin D deficiency during pregnancy is associated with unfavourable outcomes including low gestational birth weight (BW), stillbirths, preterm birth, vitamin D deficiency, hypocalcemia, and childhood obesity [6-8]. Low BW (<2500 g), stillbirth, and preterm births are birth outcomes indicative of fetal growth restrictions, while vitamin D deficiency, hypocalcemia, and chronic health outcomes suggest long-term consequences of this deficiency in later development. These outcomes suggest a threshold amount of UV exposure may be critical for healthy offspring development.

Although no international or national guidelines exist about the amount of UV or sunlight exposure preg-
This is an Open Access article distributed under the terms of the Creative Commons Attribution License (CC BY 4.0)

License deed can be found at: http://creativecommons.org/licenses/ by $/ 4.0 /$ https://doi.org/10.29173/hsi298 Received 26 February 2020 Accepted 1 June 2020 
nant women should have, the World Health Organization's INTERSUN Programme developed in 1992 remains one of the first international standards to include UV exposure as a health consideration [9]. However, it is limited and only provides general recommendations to the public to prevent adverse health risks of UV exposure. Clinical trials suggest vitamin D supplementation is ineffective in reducing longterm risks in offspring associated with maternal vitamin D deficiency [10]. Moreover, using supplementation could have availability, cost-effectiveness, and ethical (vegetarian or vegan diets) issues. As production of vitamin $\mathrm{D}$ depends primarily on exposure to sunlight [11], there might be therapeutic value in considering effects of UV exposure during pregnancy on offspring outcomes.

The objective of this commentary was to review current knowledge to inform policies and considerations for pregnant women living in Canada and regions with lower exposure to UV. This article aimed to (i) examine existing literature on the effects of sunlight exposure during pregnancy on immediate neonatal and long-term health outcomes, and (ii) formulate considerations for therapeutics and public health policy based on current evidence. We searched databases including PubMed/MEDLINE, EMBASE and Google Scholar, and no time, setting, or language restrictions were imposed on the search strategy. Primary research articles such as case studies, systematic reviews and meta-analyses, were included. Studies concerning maternal outcomes and animal studies were excluded. We hypothesize that low sunlight exposure during pregnancy is associated with adverse fetal growth-related birth outcomes and long-term disease outcomes. As a result, we anticipate a public health benefit in implementing regional health policy recommendations.

\section{UV exposure and fetal growth-related birth outcomes}

Several studies around the world have considered indicators of fetal development such as BW, preterm births and gestational size, which are common metrics for fetal growth. In a New Zealand study by Tustin et al. looking at seasonal variations over a 5-year period, exposure to bright sunlight during the first trimester was confirmed to increase BW [12]. Another New Zealand study determined prenatal seasonal peak exposures to sunlight was associated with increased BW and heights [13]. Both of these studies used a robust methodology in measuring meteorological data, based on intensity and duration of exposures specific to maternal residence, and adjusting for behavioral factors, such as smoking during pregnancy. A longitudinal study in Norway suggested UV-induced maternal vitamin $\mathrm{D}$ during pregnancy is essential for improved BW outcomes by determining increased vitamin D deficiency prevalence in pregnant women living in northern latitudes with lower exposure to sunlight [14]. Although pregnancies during darker seasons were associated with poorer outcomes in this study, this study is limited as there were no other exposure measurements. More recently however, Zhang and colleagues confirmed this trend in the first study of its kind in China, determining prenatal sunlight exposure was associated with healthy BW and reduced incidence of small for gestational age infants [15]. In particular, this study demonstrated prenatal exposure to increasing levels of sunlight exposure in the second trimester of pregnancy was most strongly associated with these positive fetal growth outcomes.

In contrast, a cross-sectional study in America conducted by Thayer determined that average annual UV index positively correlated with low BW [16]. However, the analysis revealed both racial differences and income disparities had a strong association with differences observed with low BW outcomes, suggesting these factors are effect modifiers (i.e., lower income families generally had low BW outcomes independent of UV exposure). The association between preterm birth rates and the average annual UV index were correlated revealing a greater incidence of preterm births with increasing exposures. However, a strong association with poverty factors was also seen. This association was similar to BW trends observed in this study, which utilized the same method of data collection, suggesting it had similar limitations.

Other studies suggested no association between sunlight exposure and BW outcomes in newborn infants [17-19]. Pereira et al. considered the odds ratio of small for gestational age (BW $<10^{\text {th }}$ centile for gestational week) and proportion of optimal BW in 140,000 births in Western Australia [15], while Elter et al. compared individual BW to mean BW for 3,333 births in Turkey; both studies observed no significant effects [17]. In both studies, sunlight hours were used as exposure measures and statistical analysis adjusted for meteorological and pregnancy-related variables. A recent study examined vitamin D intake through sunlight and neonatal outcomes including BW in an African population in Ghana [19], using structured questionnaires for exposure assessment. This study reported no difference when recorded sunlight exposure was compared to gestational age and Apgar scores, which are used as a measure of the infants' general health status and responsiveness immediately at birth [19]. As year-round sunlight duration and intensities are consistently high in Ghana [20], homogeneity of exposures in this particular ethnic group or region could explain the lack of difference. While UV exposure appears to be independent of these outcomes, the utilization of self-reports for exposure measurements could increase the risk of subjective reporting bias and is a limitation of this study.

\section{UV exposure and long-term conditions and disease outcomes}

When compared to birth outcomes associated with UV exposure, literature considering long-term outcomes of UV exposure during pregnancy is more limited. Despite this, there have been several original studies that examine association between UV radiation and the incidence of multiple sclerosis (MS) in offspring. In the longitudinal study conducted in 1,524 participants identified from an Australian birth regis- 
tration database, lower exposures to ambient UV during the first trimester of pregnancy was associated with an increased risk of MS in offspring [21]. The authors suggested vitamin $\mathrm{D}$ may be implicated in genetic mechanisms, which increase interactions with a locus determining susceptibility to MS. This was also considered by Vio Streym and colleagues, who used the national register database to analyze birth cohorts in Denmark to identify associations between seasonal exposures and long-term conditions including MS, type 1 diabetes, cancer, schizophrenia, ischemic heart disease and pneumonia [22]. The findings of this study demonstrated that incidence of MS and pneumonia in young subjects depended on their season of birth, suggesting low sunlight exposure in the winter months resulted in low maternal vitamin D levels during pregnancy.

Moreover, Wernerfelt et al. performed both a cross-sectional and a retrospective cohort study in the United States, examining the effects of sunlight exposure during pregnancy and the probability of asthma incidence in the offspring [23]. In the first cross-sectional study, data was collected from 264,701 individuals who responded to the 1997-2008 National Health Interview Survey regarding self-reported asthma condition, year of birth, region of residence and was associated with hours of sunlight exposure based on historical meteorological data obtained from weather stations. Although this study used surveys subjecting the data to reporter bias, it enabled the measurement of key behavioral data such as smoking, time spent outside, etc. The second retrospective cohort study created birth month-region-year cohorts and evaluated them using national hospital discharge data to determine asthma status, while sunlight exposure data was obtained from a national database. Both studies accordantly demonstrated that maternal exposure to sunlight, notably in the second trimester of pregnancy, lowers the risk for the development of asthma later in life.

Additionally, one prospective cohort study conducted by Sayers and Tobias suggests UV exposure is implicated in skeletal development during childhood [24]. This study examined 6,995 children in a Finnish cohort after UV exposure in the third trimester of pregnancy, based on meteorological monitoring data, and demonstrated a positive relationship with bone mass and size in later childhood. These findings suggest vitamin $\mathrm{D}$ levels during pregnancy have direct effects on fetal periosteal bone formation. In the study performed by Waldie and colleagues, follow-up was performed on infants of the maternal birth cohorts at regular intervals until the age of 26 and it was similarly determined that the prenatal duration of exposure to sunlight differentially influenced participant stature depending on age. However, it is notable that none of these studies made adjustments for environmental, genetic, or social factors in their analysis and this may have an impact on the validity of these results.

\section{Considerations by stages of pregnancy}

According to the developmental origins of health and disease hypothesis [3], environmental conditions during fetal development ultimately determine the health and burden of disease in the later stages of life. The majority of the evidence considered thus far suggests UV exposure is critical for different windows of vulnerability throughout fetal development. The first trimester is when the formation of the nervous system occurs [25]. It is possible that UV exposure interventions could be utilized in order to reduce risk of developing diseases or long-term conditions, such as MS, in later childhood or adulthood.

Additionally, reported associations between timing of exposures and positive birth outcomes, suggest these interventions could be useful in the second and third trimester. The second trimester marks the beginning of lung development and production of surfactant [26]. The findings made in the studies conducted by Wernerfelt et al. suggest sunlight exposure during the second trimester could lead to a reduced risk of developing asthma in later life [23]. This is conceivable as surfactant dysfunction is a risk factor for chronic airway inflammation, a signature of asthma. Similarly, interventions in the third trimester maybe essential to lowering the risk of pneumonia [22], as it is the stage when key developmental events for normal lung development occur. Larger BW outcomes could also suggest UV-induced vitamin D is implicated in normal fetal growth. There were also effects described in the third trimester [24], when skeletal development is occurring in the fetus and the presence of UV-induced maternal vitamin $\mathrm{D}$ could be required. By determining these time points, it may be possible to target sunlight exposures, particularly in mothers whose gestation overlaps with months where there is a lower exposure to sunlight.

\section{Considerations by mechanism}

With exposure to sunlight, UV radiation-induced maternal vitamin D synthesis was not the only possible mechanism for fetal changes. Tustin et al. suggested sunlight exposure early in gestation increased levels of insulin-like growth factor (IGF)-1, a hormone involved in bone and tissue development, to facilitate fetal growth [12]. Furthermore, Waldie et al. speculated maternal melatonin production was inhibited by sunlight, resulting in increased levels of growth hormone $(\mathrm{GH})$, a hormone similar to IGF-1 involved in growth and metabolism, which could also progress fetal growth [13]. These proposals may suggest the involvement of a pathway associated with melatonin production, which is critical in regulating circadian rhythm and sleep-wake cycle signaling, in fetal growth and development. This melatonin-dependent pathway may be independent or interact with vitamin $\mathrm{D}$ to increase GH/IGF-1 production.

Zhang et al. proposes the involvement of a UV radiation-dependent vitamin B9 depletion pathway [15]. Vitamin B9 consists of different forms including folate and folic acid, 
which are critical for cell growth. Not only has it been shown that UV radiation can lower concentrations of folate in the bloodstream, it is well established that folic acid deficiency during pregnancy leads to neural tube defects and resulting adverse birth outcomes $[27,28]$.

Although it is yet to be investigated, it is important to consider another mechanism of action might involve UV and sunlight-induced production of maternal serotonin. Serotonin is a neurotransmitter and hormone known to be involved in fetal brain development; several studies suggest the use of serotonin reuptake inhibitors during pregnancy to be linked with an increased incidence of autism spectrum disorder after birth [29,30]. Moreover, in conditions like seasonal affective disorder, seasonal changes, including lower amounts of sunlight and cold weather, could deplete levels of serotonin in the brain leading to depression and other psychological effects. Maternal depression is a known risk factor for detrimental socio-emotional and cognitive development of children and depression in adulthood [31,32]. It is therefore possible for birth outcomes to depend on the action of sunlight and/or UV exposure to act on any or a number of these pathways. Future studies exploring these pathways are warranted to gain more insights for the development of alternative therapeutic strategies during pregnancy, especially for women living in regions where access to sunlight is limited.

\section{Epidemiological considerations}

Epidemiological factors such as environment, time, and demographics must also be considered. While most of the original studies have been performed in industrialized countries in both the Northern and Southern Hemispheres, we determined that the numbers of studies performed in developing regions of the world on UV exposure are vastly limited, and to date, no studies have investigated the association between sunlight exposure during pregnancy and offspring outcomes in Canada. Meteorological differences in Northern continents can affect levels of exposure received, when compared to Southern latitude countries. In fact, Canadian cities, such as Iqaluit, have average hours of sunlight reach lows of 0.4 hours per day as well as an average UV index of 0 in the winter months [33]. There are also racial, socioeconomic, and geographical differences, as observed by Thayer et al. [16]. In this study, in addition to higher birth outcomes in those living in the North versus the South, there was a lower incidence of low BW and prematurity observed for non-Hispanic black women compared to non-Hispanic Caucasian women. Ngueta et al. hypothesize that these outcomes may not directly be linked to racial differences but rely instead on the closely associated maternal fat mass [34]. According to this hypothesis, there is a lower bioavailability of vitamin $\mathrm{D}$ due to its lipophilicity, which causes it to be trapped in fat masses and unable to act to prevent adverse birth outcomes.

Nonetheless, these individual-level differences are particularly important in multicultural and diverse countries such as Canada, where policymakers must take into consideration social determinants of health. Moreover, with varying exposures to sunlight in different regions coupled with differing methodologies to measure exposure effects in settings with a range of resource availabilities, it becomes difficult to make inferences about UV exposure without intricate epidemiological analysis. With genetic differences between ethnicities, individual behavioral differences, and distinctive exposure responses and capacities, another layer of complexity is added and must be accounted for when designing public health policies and recommendations. This also suggests interventions must be more personalized rather than assuming a "one-size fits all" strategy.

\section{Practical considerations for policy implementation}

Before policy implementation, further clinical trials comparing UV exposure to vitamin D supplementation for pregnant women with vitamin D deficiency are imperative. Though limited in terms of regional representation and consistent methodology, current literature suggests a therapeutic benefit in implementing health recommendation guidelines for sunlight exposure during pregnancy. Moreover, existing evidence suggests the level of benefit depends on various genetic, environmental, and behavioral factors. As a result, it is necessary for policy recommendations to be developed after consultation with an interdisciplinary collaborative of atmospheric science experts, healthcare professionals, medical scientists, public health officials and policymakers. Due to geographical variations in sunlight exposure, recommendations should be designed at the provincial or regional level considering for the intensity, duration, and other anticipated meteorological factors. Existing regional policies on environmental exposures, such as the 2006 British Columbia Reproductive Care Program Guideline for Tobacco Use in the Perinatal Period [35], which provides recommendations as the current standard for prevention and management of tobacco use and second-hand smoke exposure during pregnancy, can be used as a guide in developing novel recommendations for sunlight exposure.

\section{Conclusions}

Though scarce, the majority of available evidence suggests low sunlight exposure is associated with adverse fetal growth-related birth outcomes and long-term health outcomes. One study showed a conflicting association between sunlight exposures and birth outcomes, while two other studies demonstrated none. Exposure was implicated in long-term health outcomes including skeletal system development and reducing the incidence of MS, pneumonia and asthma after birth. This work has summarized and evaluated the main findings of the current body of evidence, identifying key limitations and implications to inform considerations for public health policy. In particular, we recognized limitations of comparing findings obtained through diverse methodologies and variable factors (i.e., geography, racial differences, inherent genetic variations, 
Table 1 | Summary of existing literature on the effects of ultraviolet exposure on fetal growth-related, neonatal and long-term outcomes. Studies identified using the search strategy presented in this review are displayed. Abbreviations: UV, Ultraviolet radiation; IGF-1, insulin-like growth factor; GH, growth hormone; MS, multiple sclerosis.

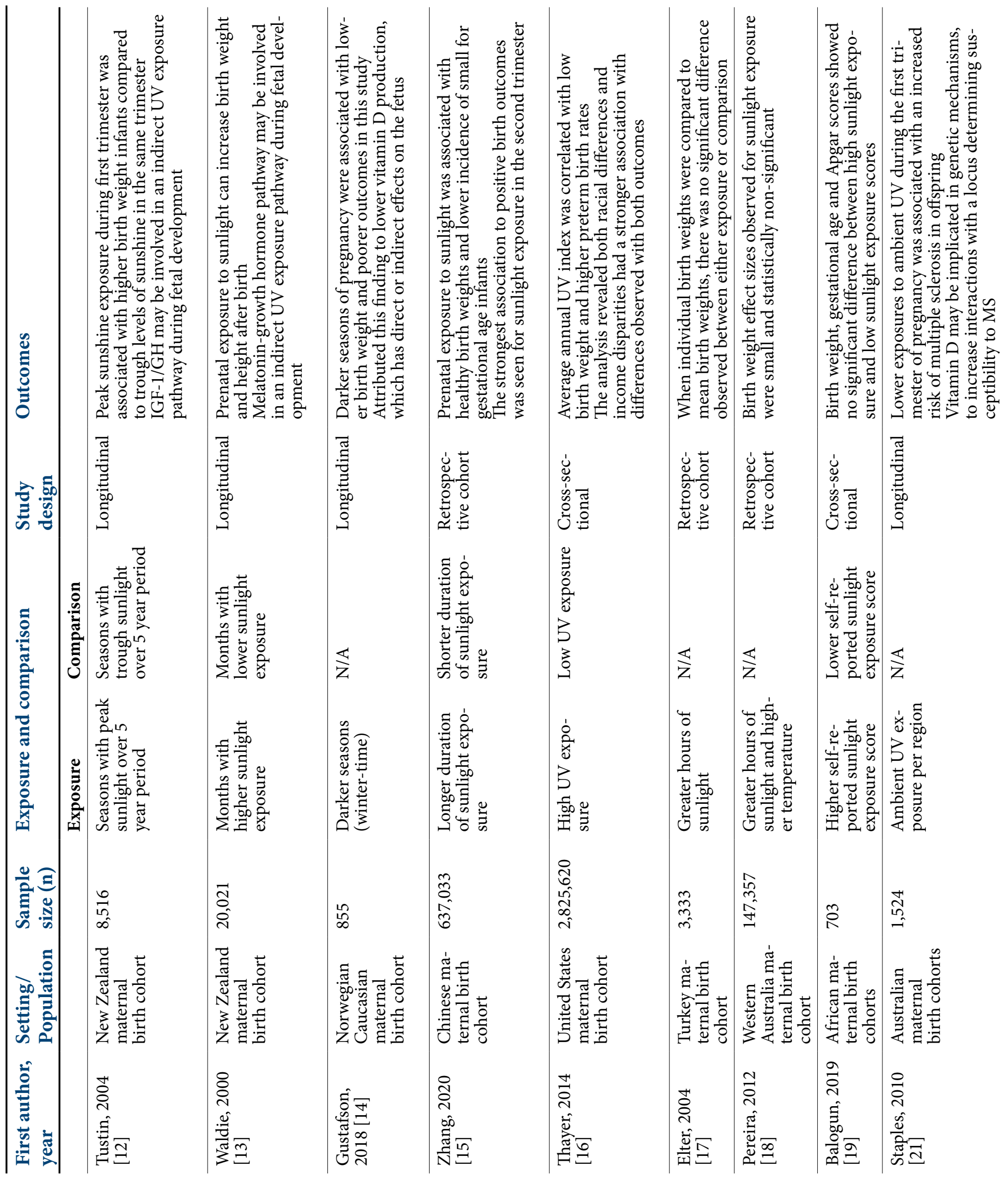


Table 1 (continued)| Summary of existing literature on the effects of ultraviolet exposure on fetal growth-related, neonatal and long-term outcomes.

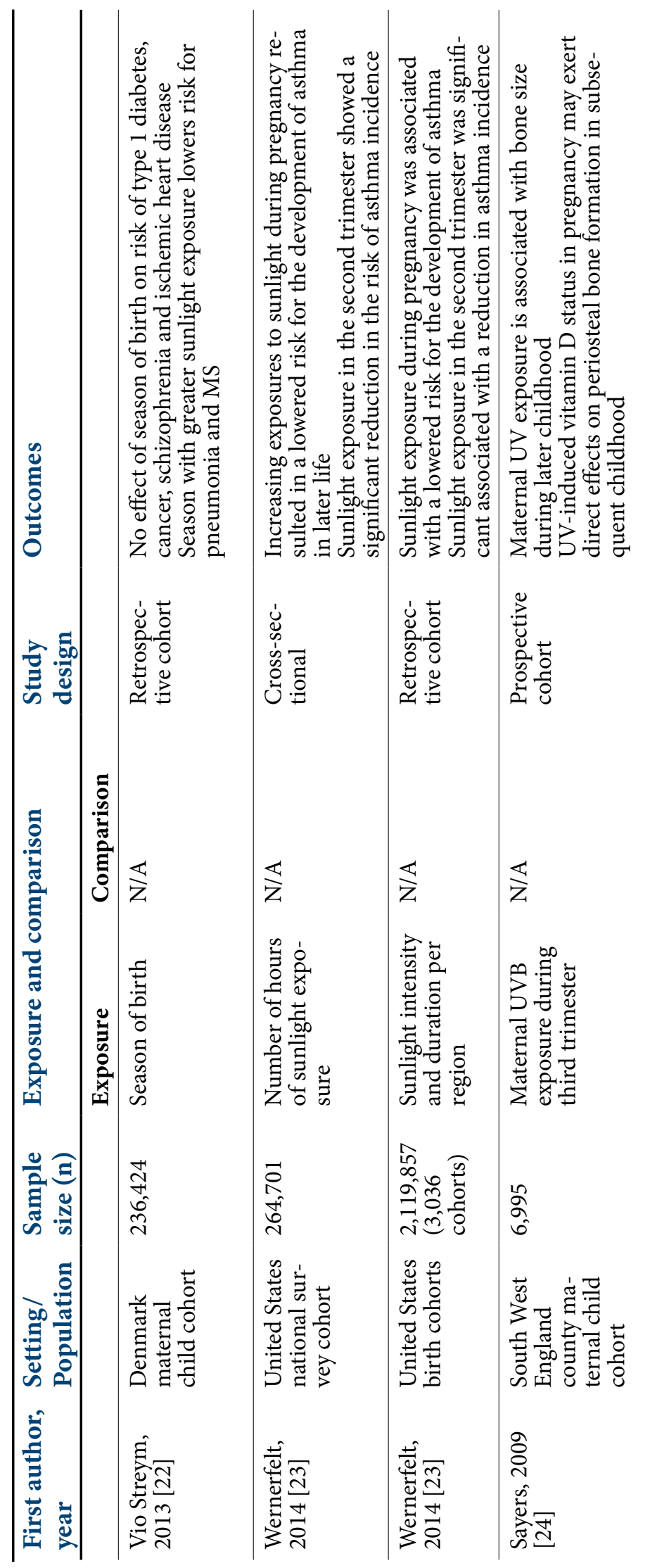

socioeconomic factors, etc.) in these studies. We have also incorporated considerations based on current understanding of exposure effect at different stages in pregnancy, different mechanisms of action, epidemiological factors, and practical considerations prior to policy implementation. Nevertheless, more research is necessary and future avenues should examine a way to quantify the threshold level of healthy exposure, personalized therapeutics to account for the effectiveness of sunlight exposure per individual, and therapeutic interventions through non-UV-vitamin D mechanisms.

\section{References}

1. United Nations Environment Programme, Environmental Effects Assessment Panel, Andrady A, Aucamp PJ, Bais AF, Ballaré CL, Björn LO, et al. Environmental effects of ozone depletion and its interactions with climate change: progress report, 2009. Photochem Photobiol Sci [Internet]. 2010 Mar [cited 2019 Mar 26];9(3):275-94. Available from: http://www.ncbi.nlm.nih.gov/pubmed/20301813

2. Gallagher RP, Lee TK, Bajdik CD, Borugian M. Ultraviolet radiation. Chronic Dis Can [Internet]. 2010 [cited 2019 Mar 26];29 Suppl 1:51-68. Available from: http://www.ncbi.nlm.nih.gov/ pubmed/21199599

3. Silveira PP, Portella AK, Goldani MZ, Barbieri MA. Developmental origins of health and disease (DOHaD). J Pediatr (Rio J). 2007 Nov;83(6):494-504.

4. Botyar M, Khoramroudi R. Ultraviolet radiation and its effects on pregnancy: A review study. J Fam Med Prim care [Internet]. 2018 [cited $2019 \mathrm{Feb} 24$ ];7(3):511-4. Available from: http://www.ncbi. nlm.nih.gov/pubmed/30112299

5. van Schoor NM, Lips P. Worldwide vitamin D status. Best Pract Res Clin Endocrinol Metab [Internet]. 2011 Aug 1 [cited 2019 Mar 26];25(4):671-80. Available from: https://www-sciencedirect-com. login.ezproxy.library.ualberta.ca/science/article/pii/S1521690X11000716 ?via\%3Dihub

6. Thorne-Lyman A, Fawzi WW. Vitamin D during pregnancy and maternal, neonatal and infant health outcomes: a systematic review and meta-analysis. Paediatr Perinat Epidemiol [Internet]. $2012 \mathrm{Jul}$ [cited 2019 Mar 26];26:75-90. Available from: http://www.ncbi.nlm. nih.gov/pubmed/22742603

7. Anastasiou A, Karras SN, Bais A, Grant WB, Kotsa K, Goulis DG. Ultraviolet radiation and effects on humans: the paradigm of maternal vitamin D production during pregnancy. Eur J Clin Nutr [Internet]. 2017 Nov 28 [cited 2019 Mar 26];71(11):1268-72. Available from: http://www.nature.com/doifinder/10.1038/ejcn.2016.188

8. Daraki V, Roumeliotaki T, Chalkiadaki G, Katrinaki M, Karachaliou $\mathrm{M}$, Leventakou V, et al. Low maternal vitamin D status in pregnancy increases the risk of childhood obesity. Pediatr Obes [Internet]. 2018 Aug 1 [cited 2019 Apr 10];13(8):467-75. Available from: http://doi. wiley.com/10.1111/ijpo.12267

9. WHO | INTERSUN Programme [Internet]. WHO. World Health Organization; 2017 [cited 2019 Mar 26]. Available from: https:// www.who.int/uv/intersunprogramme/en/

10. Roth DE, Morris SK, Zlotkin S, Gernand AD, Ahmed T, Shanta SS, et al. Vitamin D supplementation in pregnancy and lactation and infant growth. N Engl J Med. 2018 Aug 9;379(6):535-46.

11. Pérez-López FR. Low maternal vitamin $\mathrm{D}$ status during pregnancy requires appropriate therapeutic intervention. Int J Gynecol Obstet [Internet]. 2012 Jan [cited 2019 Mar 26];116(1):4-5. Available from: http://www.ncbi.nlm.nih.gov/pubmed/22036063

12. Tustin K, Gross J, Hayne H. Maternal exposure to first-trimester sunshine is associated with increased birth weight in human infants. Dev Psychobiol [Internet]. 2004 Dec [cited 2019 Mar 26];45(4):22130. Available from: http://www.ncbi.nlm.nih.gov/pubmed/15549686

13. Waldie KE, Poulton R, Kirk IJ, Silva PA. The effects of pre- and post-natal sunlight exposure on human growth: evidence from the Southern Hemisphere. Early Hum Dev [Internet]. 2000 Nov [cited 2019 Mar 26];60(1):35-42. Available from: http://www.ncbi.nlm.nih. gov/pubmed/11054582

14. Gustafsson MK, Romundstad PR, Stafne SN, Helvik A-S, Stunes 
AK, Mørkved S, et al. Alterations in the vitamin D endocrine system during pregnancy: A longitudinal study of 855 healthy Norwegian women. PLoS One [Internet]. 2018 [cited 2019 Mar 27];13(4):e0195041. Available from: http://www.ncbi.nlm.nih.gov/ pubmed/29641551

15. Zhang X, Wang Y, Chen X, Zhang X. Associations between prenatal sunshine exposure and birth outcomes in China. Sci Total Environ [Internet]. 2020 Apr 15 [cited 2020 May 1];713:136472. Available from: http://www.ncbi.nlm.nih.gov/pubmed/31955080

16. Thayer ZM. The Vitamin D hypothesis revisited: race-based disparities in birth outcomes in the United States and ultraviolet light availability. Am J Epidemiol [Internet]. 2014 Apr 15 [cited 2019 Mar 26];179(8):947-55. Available from: http://www.ncbi.nlm.nih.gov/ pubmed/24618066

17. Elter K, Ay E, Uyar E, Kavak ZN. Exposure to low outdoor temperature in the midtrimester is associated with low birth weight. Aust New Zeal J Obstet Gynaecol [Internet]. 2004 Dec [cited 2019 Mar 26];44(6):553-7. Available from: http://www.ncbi.nlm.nih.gov/ pubmed/15598296

18. Pereira G, Cook A, Haggar F, Bower C, Nassar N. Seasonal variation in fetal growth: accounting for sociodemographic, biological, and environmental exposures. Am J Obstet Gynecol [Internet]. 2012 Jan [cited 2019 Mar 26];206(1):74.e1-74.e7. Available from: http://www. ncbi.nlm.nih.gov/pubmed/21982022

19. Balogun H, Jaakkola JJK, Amegah AK. Association of sunlight exposure and consumption of Vitamin D-rich foods during pregnancy with adverse birth outcomes in an African population. J Trop Pediatr [Internet]. 2019 Jan 27 [cited 2019 Mar 26]; Available from: https://academic.oup.com/tropej/advance-article/doi/10.1093/ tropej/fmz001/5303237

20. Solargis. Solar resource maps of Ghana [Internet]. Global Solar Atlas. 2019 [cited 2020 May 4]. Available from: https://solargis.com/ maps-and-gis-data/download/ghana

21. Staples J, Ponsonby A-L, Lim L. Low maternal exposure to ultraviolet radiation in pregnancy, month of birth, and risk of multiple sclerosis in offspring: longitudinal analysis. BMJ [Internet]. 2010 Apr 29 [cited 2019 Mar 26];340:c1640. Available from: http://www. ncbi.nlm.nih.gov/pubmed/21030361

22. Við Streym S, Rejnmark L, Mosekilde L, Vestergaard P. No effect of season of birth on risk of type 1 diabetes, cancer, schizophrenia and ischemic heart disease, while some variations may be seen for pneumonia and multiple sclerosis. Dermatoendocrinol [Internet]. 2013 Apr 1 [cited 2019 Mar 27];5(2):309-16. Available from: http:// www.ncbi.nlm.nih.gov/pubmed/24194971

23. Wernerfelt N, Slusky D, Zeckhauser R, Alexander D, Bernstein A, Bleakley $\mathrm{H}$, et al. Second trimester sunlight and asthma: evidence from two independent studies [Internet]. Cambridge, MA; 2014 Oct [cited 2020 May 3]. Available from: http://www.nber.org/papers/ w20599

24. Sayers A, Tobias JH. Estimated maternal ultraviolet B exposure levels in pregnancy influence skeletal development of the child. J Clin Endocrinol Metab [Internet]. 2009 Mar [cited 2019 Mar 27];94(3):765. Available from: http://www.ncbi.nlm.nih.gov/ pubmed/19116232

25. Smith GCS. First Trimester Origins of Fetal Growth Impairment. Semin Perinatol. 2004;28(1):41-50.

26. Fox NS, Huang M, Chasen ST. Second-trimester fetal growth and the risk of poor obstetric and neonatal outcomes. Ultrasound Obstet Gynecol. 2008 Jul;32(1):61-5.

27. Fukuwatari T, Fujita M, Shibata K. Effects of UVA irradiation on the concentration of folate in human blood. Biosci Biotechnol Biochem. 2009;73(2):322-7.

28. Scholl TO, Johnson WG. Folic acid: influence on the outcome of pregnancy. . Am J Clin Nutr. 2000 Apr 30;71(5 Suppl):1295S-303S.

29. 29. Harrington RA, Lee LC, Crum RM, Zimmerman AW, Hertz-Picciotto I. Serotonin hypothesis of autism: implications for selective serotonin reuptake inhibitor use during pregnancy. Autism Res. 2013 Jun;6(3):149-68.

30. Hansson SR, Mezey É, Hoffman BJ. Serotonin transporter messenger RNA in the developing rat brain: Early expression in serotonergic neurons and transient expression in non- serotonergic neurons. Neuroscience. 1998 Jan 12;83(4):1185-201.

31. Plant DT, Pariante CM, Sharp D, Pawlby S. Maternal depression during pregnancy and offspring depression in adulthood: Role of child maltreatment. Br J Psychiatry. 2015 Sep 1;207(3):213-20.
32. Deave T, Heron J, Evans J, Emond A. The impact of maternal depression in pregnancy on early child development. BJOG An Int J Obstet Gynaecol. 2008 Jul;115(8):1043-51.

33. Environment Canada. Canadian Climate Normals 1981-2010 Station Data [Internet]. 2019 [cited 2020 May 3]. Available from: https://climate.weather.gc.ca/climate_normals/results_1981_2010_e. html? searchType $=$ stnProv\&lstProvince $=$ NU\&txtCentralLatMin $=0 \&$ txtCentralLatSec $=0 \&$ txtCentralLongMin $=0 \&$ txtCentralLongSec $=0 \& \operatorname{stnID}=1758 \&$ dispBack $=0$

34. Ngueta G, Ndjaboue R, Yepsi R. Racial difference in preterm birth and low birthweight: Towards a new hypothesis involving the interaction of 25-hydroxyvitamin D with maternal fat mass. Med Hypotheses. 2018 Dec 1;121:74-7.

35. British Columbia Reproductive Care Program. BCRP guidelines for tobacco use in the perinatal period [Internet]. Vancouver, BC; 2006 Jun [cited 2020 May 4]. Available from: http://www.perinatalservicesbc.ca/Documents/Guidelines-Standards/HealthPromotion/ TobaccoUseGuideline.pdf

(C) The Author(s) 